\title{
Do we have a Theory of Early Universe Cosmology?
}

\author{
Robert Brandenberger \\ Physics Department, McGill University, 3600 University Street, Montreal, QC, H3A \\ 2T8, Canada
}

\begin{abstract}
The inflationary scenario has become the paradigm of early universe cosmology, and - in conjuction with ideas from superstring theory - has led to speculations about an "inflationary multiverse". From a point of view of phenomenology, the inflationary universe scenario has been very successful. However, the scenario suffers from some conceptual problems, and thus it does not (yet) have the status of a solid theory. There are alternative ideas for the evolution of the very early universe which do not involve inflation but which agree with most current cosmological observations as well as inflation does. In this lecture I will outline the conceptual problems of inflation and introduce two alternative pictures - the "matter bounce" and "string gas cosmology", the latter being a realization of the "emergent universe" scenario based on some key principles of superstring theory. I will demonstrate that these two alternative pictures lead to the same predictions for the power spectrum of the observed large-scale structure and for the angular power spectrum of cosmic microwave background anisotropies as the inflationary scenario, and I will mention predictions for future observations with which the three scenarios can be observationally teased apart.
\end{abstract}

Keywords:

Early Universe, Inflationary Cosmology, Multiverse, String Gas Cosmology, Matter Bounce, Emergent Universe

\section{Introduction}

Inflationary cosmology was proposed $\mathrm{d}^{[1}$ ( work) in 1981 as a solution of some conceptual problems of the previous early universe paradigm, the Standard Big Bang model. It was soon realized ${ }^{5}$ (see also ${ }^{416}$ for related insights) that inflationary cosmology leads to the first 
causal theory for the origin of the structure in the universe which we measure today in terms of inhomogeneities in the distribution of galaxies on large scales, and anisotropies in cosmic microwave background (CMB) temperature maps. The predictions of this theory have been spectacularly confirmed by observations, in particular by the WMAP satellite results for anisotropies in the $\mathrm{CMB}^{7}$.

Inflationary cosmology has now become the paradigm of early universe cosmology. In most papers on early universe cosmology the impression is given that inflation has been firmly established and observationally proven. However, as I will argue here, this inflationary cosmology is far from proven. Although phenomenologically successful, current realizations of inflation suffer from serious conceptual problems, and hence inflationary cosmology does not (yet) have the status of an established theory. Furthermore, as realized ten years before the development of inflationary cosmology, any theory which provides an approximately scale-invariant spectrum of almost adiabatic primordial fluctuations in the early universe on scales which are larger than the Hubble radius ${ }^{1}$ will lead to inhomogeneities and anisotropies in the late time universe which are in agreement with current data ${ }^{899}$. Although inflation was the first theory based on causal physics to generate such a spectrum of fluctuations, in the mean time other scenarios have been proposed which yield the same type of spectrum. I will present two examples 2 .

In recent years, ideas from inflationary cosmology have been combined with ideas from stochastic dynamics and from string theory to generate the picture of the inflationary multiverse and the cosmological landscape. In light of the problems of inflation and other concerns which will be detailed below, I consider these ideas to be premature and warn readers (in particular those from the philosophy of physics community) not to consider them as established.

The outline of this article is as follows: I first discuss inflationary cosmology and its problems. Then, I introduce two alternative scenarios for early universe cosmology which do not involve inflation. In Section 5 (the only technical part of this article) I focus on the development of fluctuations in

\footnotetext{
${ }^{1}$ The meaning of the Hubble radius will be discussed later in the text.

${ }^{2}$ However, it must be emphasized that obtaining a scale-invariant spectrum of curvature fluctuations is by no means a general outcome of possible early universe scenarios. Pure initial vacuum and pure thermal fluctuations in standard cosmology, for example, do not lead to a scale-invariant spectrum.
} 
early universe cosmology, and I show how both inflationary cosmology and the two alternative cosmologies which I discuss yield fluctuations in agreement with current observations. Section 6 contains some concluding remarks.

\section{Review of Inflationary Cosmology}

The inflationary universe scenario is based on the assumption that there was a period in the very early universe during which space expanded almost exponentially ${ }^{3}$ (see Figure 1 for a space-time sketch of inflationary cosmology). The exponential expansion of space leads to a solution of several problems of Standard Big Bang cosmology. First of all, it leads to a horizon (defined as the forward light cone of an initial event in space-time) which is exponentially larger than it would be without the period of inflation. Provided that the period of inflation is sufficiently long, the horizon at the time $t_{r e c}$ of recombination, the time when the CMB last scattered, is larger than distance at time $t_{r e c}$ which the CMB probes. The exponential expansion of space also homogenizes the universe since the inhomogeneities are redshifted. Hence, inflation provides an explanation for the observed near isotropy of the CMB, i.e. it solves the horizon problem of Standard Cosmology. Provided that the period of inflation is longer than around $50 \mathrm{H}^{-1}$ (where $H$ is the expansion rate of space during the inflationary phase), inflation can also explain the observed spatial flatness of the universe, i.e. it solves the flatness problem. Furthermore, the exponential expansion of space explains how a Planck-scale initial universe at the Planck time can grow to encompass the entire observed universe. Related to this point, the entropy production which is a crucial aspect of inflation and occurs at the end of the inflationary phase can explain how the presently observed large entropy of the universe emerges.

Most importantly, however, inflationary cosmology provided the first causal theory for the origin of the structure in the universe which we observe in terms of inhomogeneities in the distribution of galaxies and small amplitude anisotropies in the $\mathrm{CMB}^{5}$. At the time when this was realized, no $\mathrm{CMB}$ anisotropies had been observed. It would take a decade before the initial de-

\footnotetext{
${ }^{3}$ In fact, all that is required is that the cosmological scale factor $a(t)$ is an accelerating function of time. Most models of inflation yield, however, almost exponential expansion. Hence, we will formulate the arguments in this article in the context of exponential expansion.
} 
tection $^{10}$, five more years before the characteristic oscillations in the angular power spectrum of these anisotropies were seen 11 , and five more years before the current precision maps became available ${ }^{7}$. The fact that inflationary cosmology made predictions in agreement with the high precision observations (after adjusting a small number of cosmological parameters) is a major success of the scenario. However, it is important to keep in mind that, as discussed ten years before the development of inflationary cosmology, any early universe model which generates a nearly scale-invariant spectrum of primordial almost adiabatic fluctuations will be in agreement with current observations $4^{4}$ Whereas inflationary cosmology yielded the first causal mechanism for producing this type of fluctuations, there are now several alternative scenarios, two of them which will be discussed in this lecture.

If we use Einstein gravity to describe space and time, then exponential expansion of space requires matter to be dominated by some substance with an equation of state $p=-\rho$, where $p$ and $\rho$ are pressure and energy densities, respectively. Matter which we observe in nature does not have such highly negative pressure. Even if we describe matter in terms of quantum fields (as we should since quantum field theory is the best description we have which describes matter at very high energies and thus should be used in early universe cosmology), obtaining the above equation of state requires assuming the existence of a scalar matter field $\varphi$ with a non-vanishing potential energy density $V(\varphi)$. This potential energy leads to a contribution to energy density and pressure which is in agreement with the above equation of state.

The inflationary scenario now is as follows ${ }^{12}$ : at the initial time, the scalar field is displaced from the minimum of its potential. Since the potential is tuned to be very flat, the scalar field motion is very slow. Thus, the scalar field potential energy density remains almost constant, whereas all other forms of matter redshift ${ }^{5}$. Thus, at some time $t_{i}$, the scalar field potential energy starts to dominate and inflation begins. Once the scalar field has decreased to a critical value which in many models is close to the Planck scale, the scalar field kinetic energy begins to dominate over the potential energy and inflation ends. The time when this occurs is denoted as $t_{R}$.

No fundamental particles of nature which correspond to scalar fields have yet been observed in nature. The Standard Model of particle physics does

\footnotetext{
${ }^{4}$ Scale-invariance of the fluctuation spectrum will be defined in Section 5 .

${ }^{5}$ The inflationary slow-roll trajectory is a local attractor in initial condition space ${ }^{13}$.
} 


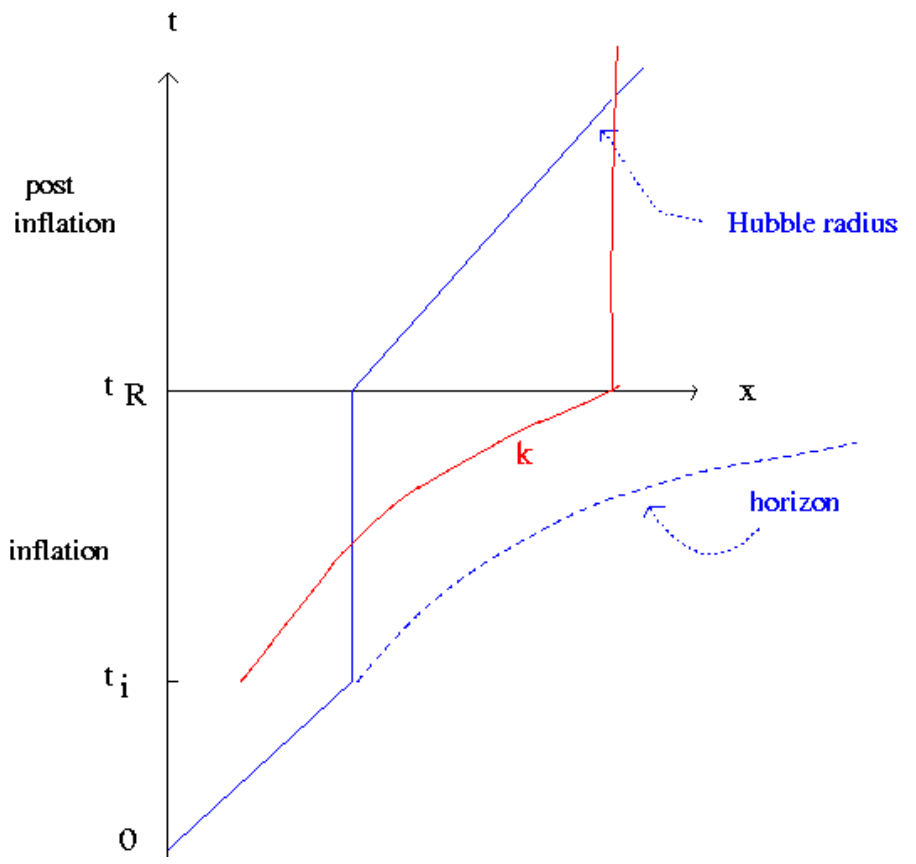

Figure 1: Space-time sketch of inflationary cosmology. The vertical axis is time, the horizontal axis corresponds to physical distance. The solid line labelled $k$ is the physical length of a fixed comoving fluctuation scale. The role of the Hubble radius and the horizon are discussed in the text. 
contain one such field, the Higgs field, the field responsible for giving the particles we observe masses. Tantalizing evidence for the existence of the Higgs field is emerging from the recent experiments at CERN, but the evidence is not yet strong enough to claim a discovery. Unfortunately, the potential of the Higgs field has the wrong shape and cannot yield cosmological inflation, unless a very unusual coupling of this field to gravity is assumed ${ }^{14}$. The scalar field potential must be tuned to be very flat in order to allow for a long period of time during which the potential energy of the field dominates over other forms.

Theories beyond the Standard Model of particle physics often contain scalar fields. In particular, supersymmetric theories have scalar field partners to all fundamental fermionic fields of spin $1 / 2$. However, to obtain the required flat potentials for these scalar fields requires fine-tuning. Thus, although cosmologists now have many toy models for inflation, there is no convincing realization of the scenario. There are also attempts to obtain inflation from modified theories of gravity rather than by introducing new forms of matter. In fact, the first model of exponential expansion of space ${ }^{3}$ was based on a modified gravity model. However, also in this context inflation does not seem to emerge in a natural way.

In spite of the lack of a firmly established theory of inflation, inflationary model building has become an industry (see e.g. 15 for reviews). Stochastic effects due to quantum fluctuations have been added ${ }^{16}$ to the classical scalar field evolution, leading to the conclusion that in some regions of field space there is a finite probability that the scalar field will move up the potential rather than down as it does with classical dynamics alone. This gives rise to a scenario ${ }^{17}$ in which inflation is eternal into the future: most of the physical volume of space remains in a state of inflation, and only pockets of space can exit inflationary expansion and can make the transition to the Standard Big Bang phase. These are pockets of space where the scalar field value has dropped below the critical value for stochastic effects to be important. The fancy name for this scenario is the eternal self-reproducing inflationary universe.

A further twist to this scenario resulted from merging the ideas of stochastic inflation with the realization that the ground state of string theory is not unique. Physicists now talk about an enormous number of string vacua, ground states of string theory 18 . Each of these ground states may have a different value of the cosmological constant $\Lambda$, and one may appeal to anthropic arguments to claim that the universe will select the ground state with 
exactly the observed value of $\Lambda$. The fancy name for the resulting picture is the string inflationary multiverse $e^{19}$ or the string landscape.

There are a couple of points to make with respect to the above recent ideas. First of all, there is nothing special to string theory in terms of having many ground states. Imagine a geographic landscape: it will also have many local minima of the gravitational potential. Second, the current analysis of string vacua is based on quantum field theory ideas inspired by string theory, not by non-perturbative string theory. We are still lacking a non-perturbative approach to string theory which is consistent with a positive or vanishing cosmological constant. There is in fact some evidence that many ground states of a string theory-motivated effective field theory are not realizable in full string theory 20 . Thirdly, the mathematical analysis which tries to include stochastic effects and which leads to the eternal self-reproducing universe is not under good mathematical controle. In fact, back-reaction effects ${ }^{21}$ which are not included in stochastic inflation may invalidate the entire analysis.

\section{Challenges for Inflationary Cosmology}

We have already discussed the first problem of inflaton, namely the question of how to embed inflation into a natural particle physics model. If we manage to overcome this problem, a second problem immediately rears its head, namely the amplitude problem. The inflationary scenario was introduced in order to eliminate fine tuning conditions for the initial data set of cosmology, and it provides a mechanism for the origin of structure in the universe. However, in a wide class of inflationary models, obtaining the correct amplitude of the cosmological perturbations requires the introduction of a large hierarchy in scales ${ }^{22}$, and thus the fine-tuning problem returns in a different guise.

A more serious problem is the trans-Planckian problem for fluctuations ${ }^{23}$. Let us return to the space-time diagram of inflation (see Figure 2). The success of inflation at providing a causal structure formation mechanism is based on the fact that scales which are currently observed originate with a physical wavelength smaller than the Hubble radius at the beginning of the period of inflation. This typically requires inflation to last about $50 \mathrm{H}^{-1}$, where $H$ is the expansion rate during the inflationary phase. However, if the period of inflation was only a bit longer, namely longer than about $70 \mathrm{H}^{-1}$, then the wavelengths of all currently observable scales were in fact smaller than the Planck length at the initial time of inflation. The problem is that we 


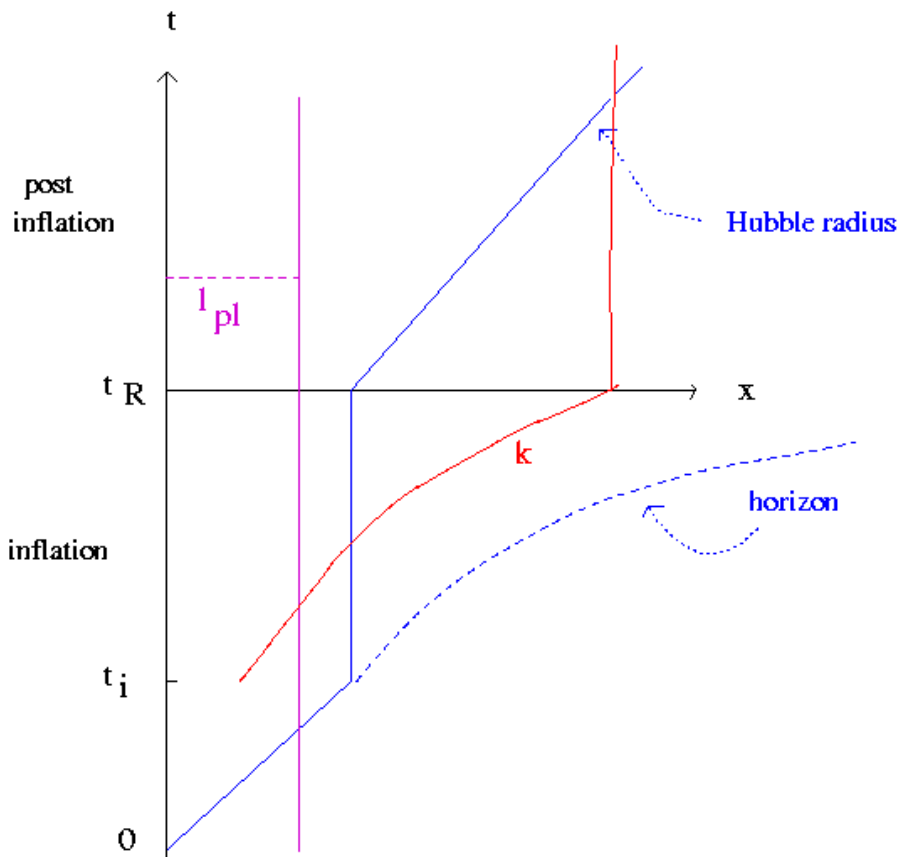

Figure 2: Space-time diagram (sketch) of inflationary cosmology where we have added an extra length scale, namely the Planck length $l_{p l}$ (majenta vertical line). The symbols have the same meaning as in Figure 2. Note, specifically, that - as long as the period of inflation lasts a couple of e-foldings longer than the minimal value required for inflation to address the problems of Standard Big Bang cosmology - all wavelengths of cosmological interest to us today start out at the beginning of the period of inflation with a wavelength which is smaller than the Planck length. 
do not know the physics operative on these scales. It is clear that both Einstein's theory of General Relativity and the theory of scalar field matter (the two ingredients on which the current theory of cosmological perturbations is based) will badly break down on these scales. Thus, the key successful prediction of inflation (the theory of the origin of fluctuations) is based on suspect calculations since new physics must enter into a correct computation of the spectrum of cosmological perturbations. The key question is as to whether the predictions obtained using the current theory are sensitive to the specifics of the unknown theory which takes over on small scales. Under certain assumptions about how physics at very high energy scales communicates with low energy physics it can be shown that the results are insensitive (see e.g. ${ }^{24}$ for an early work). However, these assumptions may not be realized in nature. In fact, simple toy models of new physics on super-Planck scales based on modified dispersion relations ${ }^{25}$ (see also ${ }^{26}$ ) or pre-inflationary dynamics 27 show that the resulting spectrum of cosmological fluctuations depends in an important way on what is assumed about physics on trans-Planckian scales. If the inflationary phase lasts for less than $70 \mathrm{H}^{-1}$, then the trans-Planckian uncertainties only effect fluctuations on smaller length scales (length scales on which the structure of the universe today is non-linear) and thus they are harder to test in cosmological precision experiments.

A fourth problem is the singularity problem. It was known for a long time that Standard Big Bang cosmology cannot be the complete story of the early universe because of the initial singularity, a singularity which is unavoidable when basing cosmology on Einstein's field equations in the presence of a matter source obeying the weak energy conditions (see e.g. ${ }^{28}$ for a textbook discussion). The singularity theorems have been generalized to apply to Einstein gravity coupled to scalar field matter, i.e. to scalar fielddriven inflationary cosmology 29 . It was shown that, in this context, a past singularity at some point in space is unavoidable. Thus we know, from the outset, that scalar field-driven inflation cannot be the ultimate theory of the very early universe.

The Achilles heel of scalar field-driven inflationary cosmology may be the cosmological constant problem. We know from observations that the large quantum vacuum energy of field theories does not gravitate today. However, to obtain a period of inflation one is using the part of the energymomentum tensor of the scalar field which looks like the vacuum energy. In the absence of a solution of the cosmological constant problem it is unclear whether scalar field-driven inflation is robust, i.e. whether the mechanism 
which renders the quantum vacuum energy gravitationally inert today will not also prevent the vacuum energy from gravitating during the period of slow-rolling of the inflaton field.

A final problem which we will mention here is the concern that the energy scale at which inflation takes place is too high to justify an effective field theory analysis based on Einstein gravity. In simple toy models of inflation, the energy scale during the period of inflation is about $10^{16} \mathrm{GeV}$, very close to the string scale in many string models, and not too far from the Planck scale. Thus, correction terms in the effective action for matter and gravity may already be important at the energy scale of inflation, and the cosmological dynamics may be rather different from what is obtained when neglecting the correction terms.

In Figure 3 we show once again the space-time sketch of inflationary cosmology. In addition to the length scales which appear in the previous versions of this figure, we have now shaded the "zones of ignorance", zones where the Einstein gravity effective action is sure to break down. As described above, fluctuations emerge from the short distance zone of ignorance (except if the period of inflation is very short), and the energy scale of inflation might put the period of inflation too close to the high energy density zone of ignorance to trust the predictions based on using the Einstein action.

Because of the above conceptual problems, the inflationary scenario may be the current paradigm of early uiverse cosmology, but it is by no means a complete theory. The problems provide a strong motivation to search for alternative early universe scenarios, two of which are described below. To be of interest, an alternative scenario should solve most of the problems of Standard Big Bang cosmology which inflation addresses, it should mitigate some of the problems of inflation, it must be consistent with the current data on the large-scale structure of the universe, and it should make some predictions with which it can be distinguished with future observations from the inflationary scenario.

\section{Two Alternative Scenarios}

\subsection{Matter Bounce}

The idea of a bouncing universe has a long history both in physics and in cosmology. In such a scenario, time is eternal both in our past and in our future. This has implications for the philosophy of cosmology: there is no mysterious creation event, and we are no longer faced with the problem - 


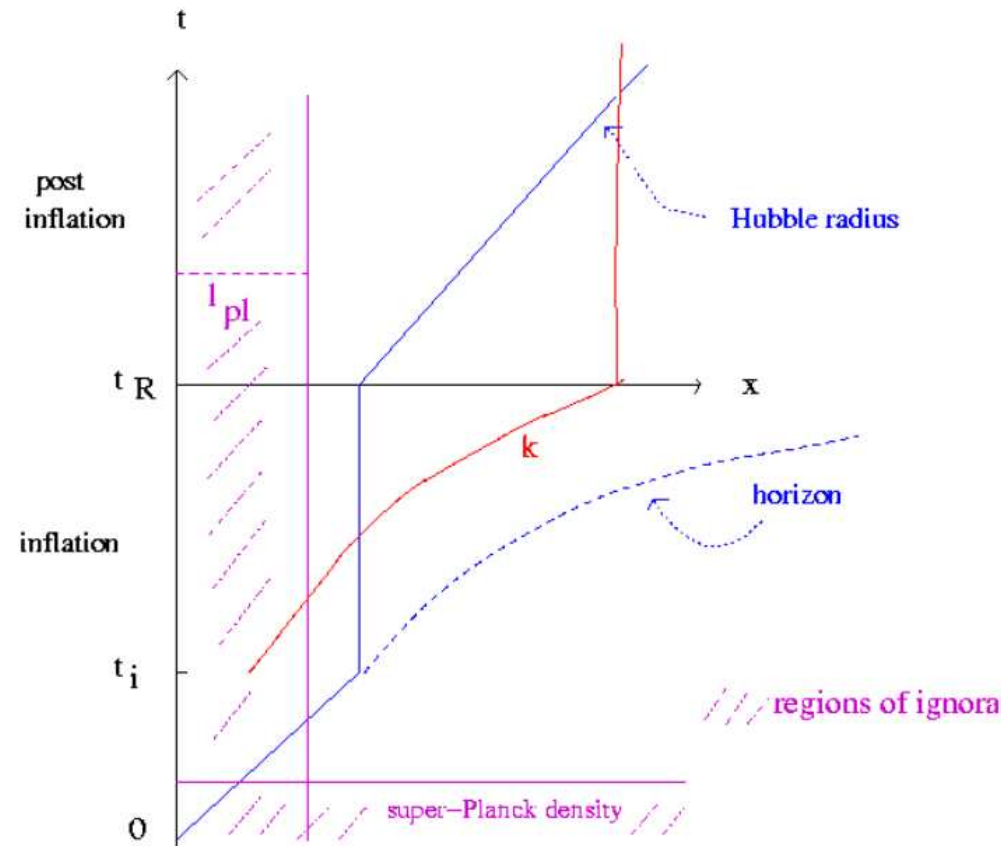

Figure 3: Space-time diagram (sketch) of inflationary cosmology including the two zones of ignorance - sub-Planckian wavelengths and trans-Planckian densities. The symbols have the same meaning as in Figure 2. Note, specifically, that - as long as the period of inflation lasts a couple of e-foldings longer than the minimal value required for inflation to address the problems of Standard Big Bang cosmology - all wavelengths of cosmological interest to us today start out at the beginning of the period of inflation with a wavelength which is in the zone of ignorance. 
insoluble in the context of pure physics - of explaining an initial singularity 6.

There have been many attempts to construct bouncing cosmologies in the context of physical cosmology (for references the reader is referred to $\stackrel{31}{31}$ ). More recently, there have been attempts to use bouncing cosmologies to provide mechanisms alternative to inflation for providing the scale-invariant spectrum of cosmological observations which observations tell us must have been generated in the early universe. The "Pre-Big-Bang" scenario ${ }^{32}$ is one attempt which was motivated from string theory dualities (similar dualities as the dualities which give rise to the "String Gas Cosmology" 33 implementation of the "emergent Universe" scenario which will be discussed in the second part of this section). A more recent construction is the "Ekpyrotic Universe" scenario $^{34}$ (and its cyclic version ${ }^{35}$ ) which was initially also motivated by superstring theory. Neither the Pre-Big-Bang nor the Ekpyrotic scenario provide a scale-invariant spectrum of adiabatic fluctuations (at least when analyzed from a purely four space-time dimensional point of view - see $e^{\sqrt{36}}$ for a higher-dimensional study), but it is not hard to find variants of the scenarios which lead to a scale-invariant spectrum of entropy fluctuations (which then later convert to adiabatic fluctuations).

I wish to discuss a simple bouncing scenario which - as realized in ${ }^{37 / 38}$ - leads to a scale-invariant spectrum of density perturbations. This is the "matter bounce" scenario. Roughly speaking, the scenario involves a contracting phase which is the time reverse of our current cosmological phase of expansion which is then smoothly connected at some high density to the expanding phase of Standard Cosmology. Obviously, new physics is required to obtain a smooth transition from contraction to expansion.

Figure 4 is a space-time sketch of the matter bounce scenario. The vertical axis is time, the horizontal axis corresponds to comoving length. As is obvious, modes which we observe today start out on sub-Hubble scales in the early phase of contraction. Hence, it is possible to have a causal mechanism for structure formation. As will be discussed in the section on cosmological perturbations, we assume that perturbations start out in their vacuum state. This is in line with the assumption that the universe started out large and almost empty. What it crucial for the success of the matter

\footnotetext{
${ }^{6}$ However, even if the creation event is absent, there are nevertheless conceptual/philosophical problems. For a discussion of this issue see e.g. $\frac{30}{\text {. }}$.
} 
bounce scenario as a theory of structure formation is that wavelengths which we observe today in cosmological observations left the Hubble radius during the matter-dominated phase of contraction. It is these scales which acquire a scale-invariant spectrum.

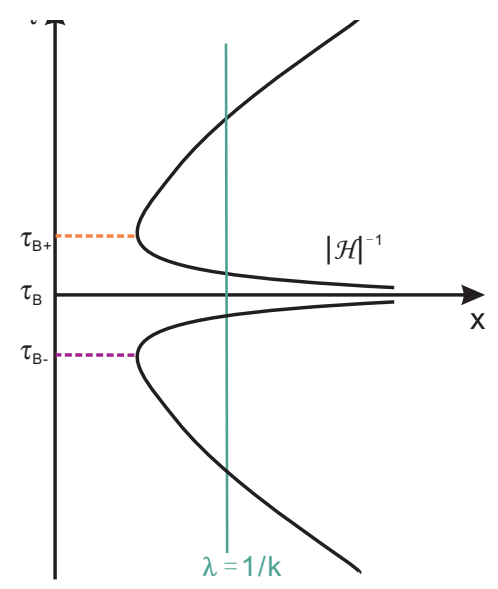

Figure 4: Space-time sketch in the matter bounce scenario. The vertical axis is conformal time $\eta$, the horizontal axis denotes a co-moving space coordinate (a co-moving coordinate grid expands as space expands like a grid painted onto the surface of a balloon expands as the balloon is inflated). The vertical line indicates the wavelength of a fluctuation mode. Also, $\mathcal{H}^{-1}$ denotes the co-moving Hubble radius.

How are the problems of Standard Big Bang cosmology (discussed at the beginning of Section 2) addressed in the matter bounce scenario? First of all, note that since the universe begins large, the size and entropy problems of Standard Cosmology do not arise. In addition, there is no horizon problem as long as the contracting period is long (to be specific, of similar duration as the post-bounce expanding phase until the present time). As already mentioned, there is a causal mechanism for generating the primordial cosmological perturbations which evolve into the structures we observe today.

The flatness problem is the one which is only partially addressed in the matter bounce setup. The contribution of the spatial curvature decreases in the contracting phase at the same rate as it increases in the expanding phase. Thus, to explain the observed spatial flatness, comparable spatial flatness at 
early times in the contracting phase is required. This is an improved situation compared to the situation in Standard Big Bang cosmology where spatial flatness is overall an unstable fixed point and hence extreme fine tuning of the initial conditions is required to explain the observed degree of flatness. But the situation is not as good as it is in a model with a long period of inflation where spatial flatness is a local attractor in initial condition space (it is not a global attractor, though!).

As any good alternative to the inflationary scenario should, the matter bounce paradigm successfully addresses some of the conceptual problems of inflation. Possibly most importantly, the length scale of fluctuations of interest for current observations on cosmological scales is many orders of magnitude larger than the Planck length throughout the evolution. If the energy scale at the bounce point is comparable to the particle physics "Grand Unified Theory" (GUT) scale, then typical wavelengths at the bounce point are not too different from $1 \mathrm{~mm}$. Hence, the fluctuations never get close to the small wavelength zone of ignorance in Figures 2 and 3, and thus a description of the evolution of fluctuations using Einstein gravity should be well justified modulo possible difficulties at the bounce point which we will return to. Thus, there is no trans-Planckian problem for fluctuations in the matter bounce scenario.

As already mentioned, new physics is required in order to provide a nonsingular bounce. Thus, the "solution" of the singularity problem is put in by hand and cannot be counted as a success, except in realizations of the matter bounce in the context of a string theory background in which the non-singular evolution follows from general principles. Such a theory has recently been presented in ${ }^{[39}$ ( $\operatorname{see}^{\sqrt{40}}$ for an analysis of fluctuations in these models). Existing matter bounce models do not address the cosmological constant problem. However, I would like to emphasize that the mechanism which drives the evolution in the matter bounce scenario is robust against our ignorance of what solves the cosmological constant problem, an improvement of the situation compared to inflationary cosmology.

However, the matter bounce scenario presented here suffers from a serious anisotropy problem. Since the energy density in anisotropies scales as $a^{-6}$, where $a(t)$ is the cosmological scale factor, and thus increases faster than that of matter (scaling as $a^{-3}$ ) and radiation (scaling as $a^{-4}$ ), it increases relative to that of matter and radiation. This endangers the smooth nearly homogeneous and isotropic bounce, unless the initial anisotropy is tuned to an extremely small value. Note that the Ekpyrotic scenario, which also 
involves a phase of contraction, is immune from this problem since the energy density in the field which is responsible for the Ekpyrotic contraction grows even faster as $a$ decreases.

With Einstein gravity and matter satisfying the usual energy conditions it is not possible to obtain a non-singular bounce. Thus, new physics is required in order to obtain a non-singular bouncing cosmology. Such new physics can arise by modifying either the gravitational or the matter sector.

To obtain a bouncing cosmology by modifying matter it is necessary to introduce a new form of matter which violates the Null Energy Condition (NEC), a condition which prohibits negative energy densities. It is necessary to arrange this new form of matter such that it dominates only at very high densities. The simplest way to obtain a bounce is to use two forms of matter, the first regular matter, and the second a "quintom field" 41 whose contributions to the energy density and pressure are opposite to that of regular matter. The universe starts out in a contracting phase dominated by regular matter. The dynamics of the quintom field is then arranged in a way such that at high energy densities the absolute value of the energy density in the quintom field increases compared to that in regular matter. The total energy density then decreases to zero, allowing for a cosmological bounce to occur ${ }^{42}$ (some important details are omitted in this discussion, and the interested reader is referred to ${ }^{43}$ for details). This simplest way of obtaining a cosmological bounce is plagued by an instability of the vacuum ${ }^{44}$. More sophisticated models which avoid this instability have recently been developed, e.g. the ghost condensate scenario ${ }^{45 / 46}$ or the Galileon bounce ${ }^{47}$. However, these models do not (yet) come from a theory of matter and gravity which is complete at high energies.

Possibly a more promising approach to obtaining bouncing cosmologies is by modifying the gravitational sector of the theory. There is excellent motivation to consider modifications of gravity at high energy densities: General Relativity is not a renormalizable quantum theory of gravity. In all known approaches to quantum gravity, the Einstein action of General Relativity is only a low energy effective action. At high energy densities where the bounce is expected to occur deviations from General Relativity will be important. Two examples of modifications of General Relativity at high densities which lead to bouncing cosmologies are the "nonsingular universe" construction of ${ }^{48}$ and the ghost-free higher derivative action of ${ }^{49}$. It was also realized ${ }^{50}$ that the Hořava-Lifshitz proposal for a power-counting renormalizable theory of quantum gravity ${ }^{51}$ leads to a bouncing homogeneous and isotropic 


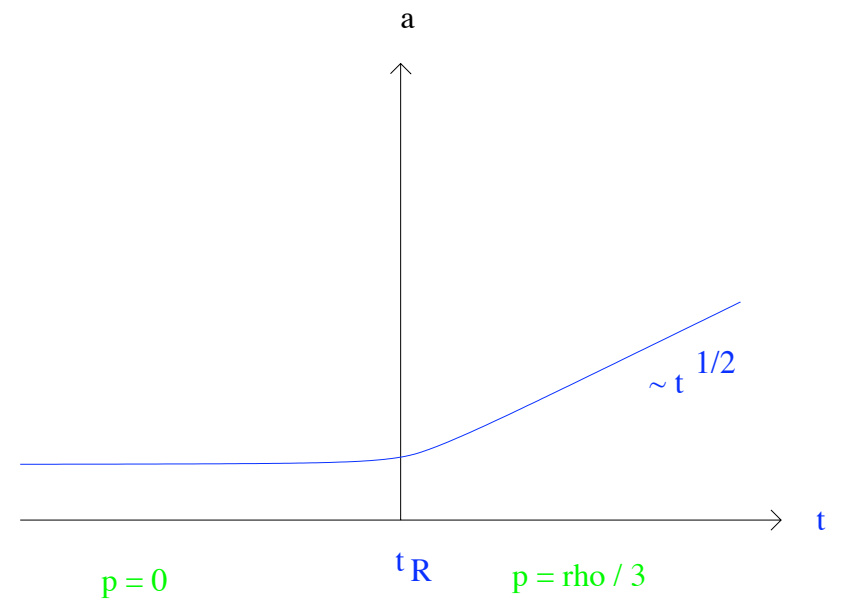

Figure 5: The dynamics of emergent universe cosmology. The vertical axis represents the scale factor of the universe, the horizontal axis is time.

cosmology, provided that the spatial curvature is non-vanishing.

Bouncing cosmologies also are predicted in more ambitious approaches to quantizing gravity such as string theory (see e.g. ${ }^{39}$ ) and loop quantum cosmology (see e.g. ${ }^{[52}$ for recent reviews).

\subsection{Emergent Universe}

The "emergent universe" scenario 53 is another non-singular cosmological scenario in which time runs from $-\infty$ to $+\infty$. The universe is assumed to emerge in a quasi-static high density phase which at some time (which is conventionally called $t=0$ undergoes a phase transition to the expanding phase of Standard Big Bang cosmology. The time evolution of the scale factor is sketched in Figure 5. For the purposes of establishing a theory of cosmological structure formation, the quasi-static phase is not required to be infinite. All that is required is that the phase is much longer than the length which in the expanding phase grows to become the current Hubble radius. The quasi-static phase could thus be the bounce phase of a bouncing cosmology, as in the model of ${ }^{54}$ (which is based on the higher derivative gravitational Lagrangian of $\frac{49}{4}$.

The time evolution of the cosmological scale factor in an emergent universe is sketched in Fig. 5. The vertical axis is the cosmological scale factor, the horizontal axis is time. The universe is initially static and makes a smooth transition to the radiation phase of Standard Big Bang cosmology. 


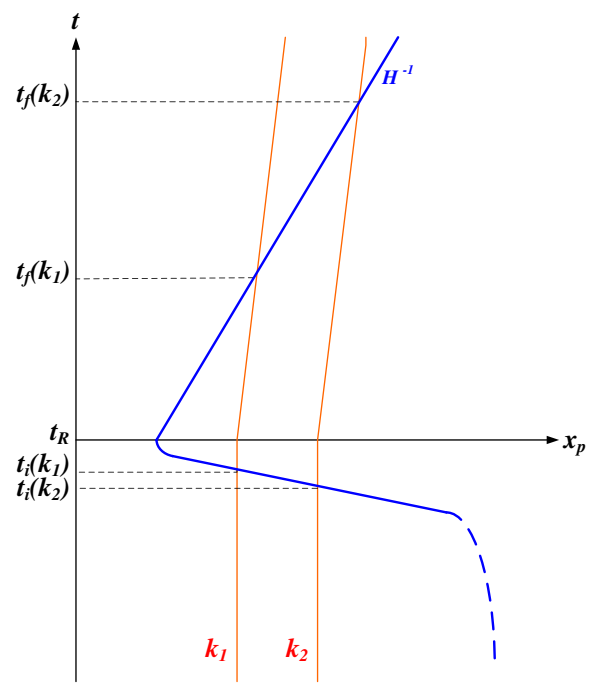

Figure 6: Space-time diagram (sketch) showing the evolution of fixed co-moving scales in emergent cosmology. The vertical axis is time, the horizontal axis is physical distance. The solid curve represents the Hubble radius $H^{-1}$ which shrinks abruptly to a micro-physical scale at $t_{R}$ and then increases linearly in time for $t>t_{R}$. Fixed co-moving scales (the dotted lines labeled by $k_{1}$ and $k_{2}$ ) which are currently probed in cosmological observations have wavelengths which were smaller than the Hubble radius long before $t_{R}$. They exit the Hubble radius at times $t_{i}(k)$ just prior to $t_{R}$, and propagate with a wavelength larger than the Hubble radius until they re-enter the Hubble radius at times $t_{f}(k)$.

The emergent scenario is similar to inflationary cosmology in that the universe is assumed to begin hot and small. But it is similar to a bouncing cosmology in that time runs from $-\infty$ to $+\infty$, and in that the evolution is non-singular.

In Figure 6 we sketch the space-time diagram in an emergent cosmology. Since the early emergent phase is quasi-static, the Hubble radius is infinite. For the same reason, the physical wavelength of fluctuations remains constant in this phase. At the end of the emergent phase, the Hubble radius decreases to a microscopic value and makes a transition to its evolution in Standard Cosmology.

As in inflationary cosmology and in a bouncing cosmology we see that 
fluctuations originate on sub-Hubble scales. In emergent cosmology, it is the existence of a quasi-static phase which leads to this result. What sources fluctuations depends on the realization of the emergent scenario. String Gas Cosmology is the example which I will consider later on. In this case, the source of perturbations is thermal: string thermodynamical fluctuations in a compact space with stable winding modes, and this in fact leads to a scaleinvariant spectrum ${ }^{55}$.

How does emergent cosmology address the problems of Standard Cosmology? As in the case of a bouncing cosmology, the horizon is infinite and hence there is no horizon problem. Since there is likely thermal equilibrium in the emergent phase, a mechanism to homogenize the universe exists. As discussed in the previous paragraph, there is no causality obstacle against producing cosmological fluctuations. The scenario is non-singular, but this cannot in general be weighted as a success unless the emergent phase can be shown to arise from some well controlled ultraviolet physics.

Like in the case of a bouncing cosmology, there is no trans-Planckian problem for fluctuations - their wavelength never gets close to the Planck scale. And like in the case of a bouncing cosmology, the physics driving the background dynamics is robust against our ignorance of what solves the cosmological constant problem. These are two advantages of the emergent scenario compared to inflation.

On the negative side, the origin of the large size and entropy of our universe remains a mystery in emergent cosmology. Also, the physics yielding the emergent phase is not well understood in terms of an effective field theory setting, in contrast to the physics yielding inflation.

String gas cosmology ${ }^{\sqrt{33}}$ (see also ${ }^{56}$, and see ${ }^{57 / 58}$ for a comprehensive review) is a specific realization of the emergent universe paradigm. It is a toy model of cosmology which results from coupling a gas of fundamental strings to a background space-time metric. The idea is to study consequences for cosmology of some of the basic principles which distinguish string theory from point particle theories. String theory contains new degrees of freedom, and this leads to new symmetries. These new degrees of freedom and new symmetries will lead to an evolution of the early universe which is profoundly different from what can be obtained if one works in the restricted context of point particle theories only.

Let us be a bit more specific. First of all, it is assumed that the spatial sections are compact. For simplicity, each spatial direction can be taken to be a circle with radius $R$. Strings have three types of states: momentum 


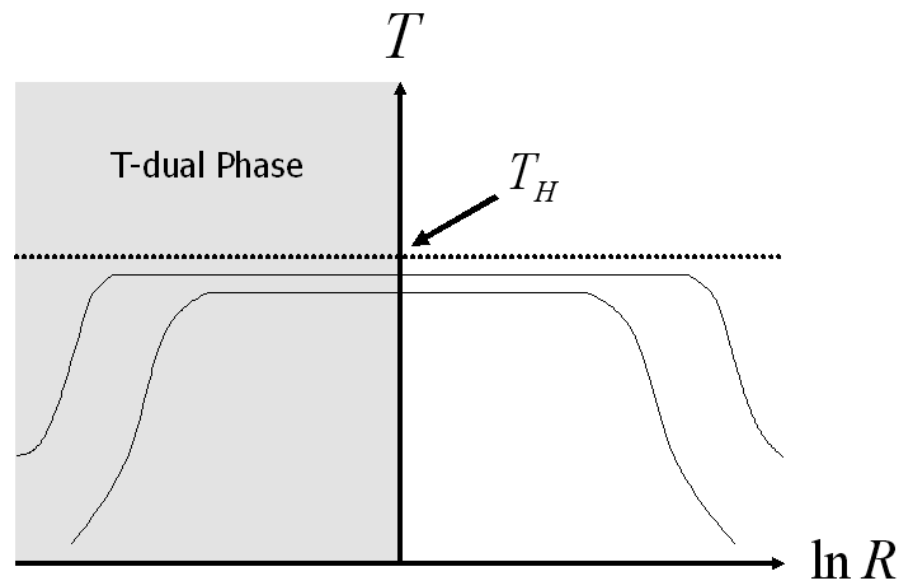

Figure 7: The temperature (vertical axis) as a function of radius (horizontal axis) of a gas of closed strings in thermal equilibrium. Note the absence of a temperature singularity. The range of values of $R$ for which the temperature is close to the Hagedorn temperature $T_{H}$ depends on the total entropy of the universe. The upper of the two curves corresponds to a universe with larger entropy.

modes which represent the center of mass motion of the string, oscillatory modes which represent the fluctuations of the strings, and winding modes counting the number of times a string wraps the spatial circle. Oscillatory and winding modes are not present in point particle theories. Both lead to important consequences in early universe cosmology.

Since the number of string oscillatory states increases exponentially with energy, there is a limiting temperature for a gas of strings in thermal equilibrium, the Hagedorn temperature ${ }^{59} T_{H}$. Thus, if we take a box of strings and adiabatically decrease the box size, the temperature will never diverge. This is the first indication that string theory has the potential to resolve the cosmological singularity problem. Figure (7) is a sketch of how the temperature of a gas of strings depends on the radius of space.

The second key feature of string theory upon which string gas cosmology is based is T-duality. To introduce this symmetry, let us discuss the radius dependence of the energy of the basic string states: The energy of an oscillatory mode is independent of $R$, momentum mode energies are quantized in units of $1 / R$, i.e.

$$
E_{n}=n \mu \frac{l_{s}^{2}}{R}
$$


where $l_{s}$ is the string length and $\mu$ is the mass per unit length of a string. The winding mode energies are quantized in units of $R$, i.e.

$$
E_{m}=m \mu R,
$$

where both $n$ and $m$ are integers. Thus, a new symmetry of the spectrum of string states emerges: Under the change

$$
R \rightarrow 1 / R
$$

in the radius of the torus (in units of $l_{s}$ ) the energy spectrum of string states is invariant if winding and momentum quantum numbers are interchanged. Perturbative string interactions are consistent with this symmetry, and thus T-duality is a symmetry of perturbative string theory. Postulating that T-duality extends to non-perturbative string theory leads ${ }^{60}$ to the need of adding D-branes to the list of fundamental objects in string theory. With this addition, T-duality is expected to be a symmetry of non-perturbative string theory 7 . As discussed in $\frac{33}{3}$, the above T-duality symmetry leads to an equivalence between small and large spaces

Based on the above features of string theory, we can construct the string gas realization of the emergent universe paradigm in the following way: we assume that the universe starts in a quasi-static phase during which the temperature of the string gas hovers at the Hagedorn value ${ }^{59}$, the maximal temperature of a gas of closed strings in thermal equilibrium 8 . The string gas in this early phase is dominated by strings winding the compact spatial sections. The annihilation of winding strings will produce string loops 9 and lead to a transition from the early quasi-static phase to the radiation phase of Standard Cosmology.

\footnotetext{
${ }^{7}$ Specifically, T-duality will take a spectrum of stable Type IIA branes and map it into a corresponding spectrum of stable Type IIB branes with identical masses $\underline{61}$.

${ }^{8}$ The equations that govern the background of string gas cosmology are not known. The Einstein equations are not the correct equations since they do not obey the T-duality symmetry of string theory. Many early studies of string gas cosmology were based on using the dilaton gravity equations. However, these equations are not satisfactory, either. Dilaton gravity is a low energy approximation to the action of string theory which only goes slightly beyond the Einstein action. However, we expect that string theory correction terms to the low energy effective action of string theory will be dominant in the Hagedorn phase, and that leading low energy effective Lagrangians will give a very misleading description of the dynamics.

${ }^{9}$ String loops have an equation of state like that of radiation.
} 
As pointed out in 33 , the annihilation of winding strings into string loops is possible in at most three large spatial dimensions. This is a simple dimension counting argument: string world sheets have measure zero intersection probability in more than four large space-time dimensions. Hence, string gas cosmology may provide a natural mechanism for explaining why there are exactly three large spatial dimensions. Once three spatial dimensions have started to expand, the string modes winding other spatial sections cannot find eachother. Hence, the radii of the extra spatial dimensions remain at the string scale at all times. This argument was supported by numerical studies of string evolution in three and four spatial dimensions ${ }^{62}$ (see also ${ }^{63}$ ) 10. The extra spatial dimensions are confined at fixed radius and shape by the string states which have winding and momentum about them. Thus, string gas cosmology provides a natural mechanism to stabilize most of the string moduli which traditionally pose a serious problem in any attempt to merge string theory with cosmology. This issue is discussed in the original

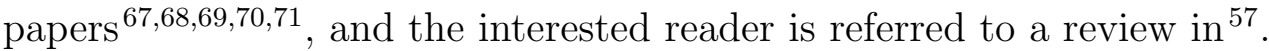

\section{Making Contact with Cosmological Observations}

Cosmology is currently in its golden ages in terms of the wealth of new observational data which are being collected every year. Thus, the main goal of modern theoretical cosmology has become making contact between early universe cosmology and the data about the structure of the observed universe. The main tool being used is the theory of cosmological perturbations. In the following I give a brief overview of this theory and demonstrate its application to both inflationary cosmology and to the two alternative cosmological scenarios which were discussed above. For a more technical overview, the reader is referred in ${ }^{72}$, and for an in-depth survey to ${ }^{73}$.

\subsection{Cosmological Perturbations}

Cosmological perturbations are inhomogeneities in both the metric of space-time and in the matter distribution. To describe the generation and evolution of these fluctuations, both General Relativity and quantum mechanics are required - General Relativity since the wavelengths of the inhomogeneities which we are interested in are larger than the Hubble radius

\footnotetext{
${ }^{10} \mathrm{See} \sqrt{64 \sqrt[65 \mid 66]{6}}$ for some caveats to this argument.
} 
11 for a long period of time, and quantum mechanics since in many models the origin of the fluctuations is quantum mechanical ${ }^{12}$. What makes the theory of cosmological perturbations tractable is that the amplitude of the fractional fluctuations is small today and hence (since gravity is a purely attractive force) that it was even smaller in the early universe. This justifies the linear analysis of the generation and evolution of fluctuations.

In the context of a Universe with an inflationary period, the quantum origin of cosmological fluctuations was first discussed in ${ }^{5}$ (see also ${ }^{\sqrt[614]{4}}$ for earlier ideas, and ${ }^{75}$ for the corresponding analysis of gravitational wave production). In particular, Mukhanov ${ }^{5}$ and Press ${ }^{\sqrt{6}}$ realized that in an exponentially expanding background, the curvature fluctuations would be scale-invariant, and Mukhanov provided a quantitative calculation which also yielded a logarithmic deviation from exact scale-invariance.

The basic idea of the theory of cosmological perturbations is simple. In order to obtain the action for linearized cosmological perturbations, we expand the action for gravity and matter to quadratic order in the fluctuating degrees of freedom. The linear terms cancel because the background is taken to satisfy the background equations of motion.

At first sight, it appears that there are ten degrees of freedom for the metric fluctuations, in addition to the matter perturbations. However, four of these degrees of freedom are equivalent to space-time diffeomorphisms. To study the remaining six degrees of freedom for metric fluctuations it proves very useful to classify them according to how they transform under spatial

\footnotetext{
${ }^{11}$ The Hubble radius is defined as $H^{-1}(t)$, where $H(t)$ is the expansion rate of space at time $t$, and it plays an important role in cosmology since it separates length scales where matter interactions dominate (sub-Hubble) from those larger ones where matter forces freeze out and gravity dominates. It is important to realize that the Hubble radius is not the same thing as the horizon, the distance which light can travel starting at the initial time. In all theories which have a chance of explaining the origin of structure, the horizon must be much larger than the Hubble radius.

${ }^{12} \mathrm{As}$ discussed in the following subsection, classical fluctuations are red-shifted during inflation, hence leaving behind a vacuum state of matter. Thus, the source of fluctuations should be from the quantum vacuum. However, if there is a mechanism which generates matter during inflation (like e.g. in the "warm inflation" scenario $\frac{74}{}$ ), or if trans-Planckian effects re-populate high energy modes, then classical (e.g. thermal) fluctuations will dominate. In some alternatives to inflation (e.g. the "matter bounce" scenario) it is postulated that the origin of the inhomogeneities in quantum mechanical, while in others (e.g. "string gas cosmology") it is thermal.
} 
rotations. There are two scalar modes, two vector modes and two tensor modes (which are the two helicity states of gravitational waves). At linear order in cosmological perturbation theory, scalar, vector and tensor modes decouple. It is the scalar fluctuations which are the most important since they describe how small matter perturbations lead to fluctuations which grow in time and can become the very inhomogeneous distribution of galaxies today. For simple forms of matter such as scalar fields or perfect fluids, the matter fluctuations couple only to the scalar metric modes. These are the so-called "cosmological perturbations" which we focus on.

If matter has no anisotropic stress, then one of the scalar metric degrees of freedom disappears. In addition, one of the Einstein constraint equations couples the remaining metric degree of freedom to matter. Thus, if there is only one matter component (e.g. one scalar matter field), there is only one independent scalar cosmological fluctuation mode.

To obtain the action and equation of motion for this mode, we begin with the Einstein-Hilbert action for gravity and the action for matter (which we take for simplicity to be a scalar field $\varphi$ - for the more complicated case of general hydrodynamical fluctuations the reader is referred to ${ }^{73}$ )

$$
S=\int d^{4} x \sqrt{-g}\left[-\frac{1}{16 \pi G} R+\frac{1}{2} \partial_{\mu} \varphi \partial^{\mu} \varphi-V(\varphi)\right]
$$

where $R$ is the Ricci curvature scalar.

The simplest way to proceed is to work in longitudinal gauge, in which the metric and matter take the form (assuming no anisotropic stress)

$$
\begin{aligned}
d s^{2} & =a^{2}(\eta)\left[(1+2 \Phi(\eta, \mathbf{x})) d \eta^{2}-(1-2 \Phi(t, \mathbf{x})) d \mathbf{x}^{2}\right] \\
\varphi(\eta, \mathbf{x}) & =\varphi_{0}(\eta)+\delta \varphi(\eta, \mathbf{x})
\end{aligned}
$$

where $\eta$ in conformal time related to the physical time $t$ via $d \eta=a(t)^{-1} d t$. The two fluctuation variables $\Phi$ and $\delta \varphi$ must be linked by the Einstein constraint equations since there cannot be matter fluctuations without induced metric fluctuations.

The two nontrivial tasks of the lengthy ${ }^{73}$ computation of the quadratic piece of the action is to find out what combination of $\delta \varphi$ and $\Phi$ gives the variable $v$ in terms of which the action has canonical kinetic term, and what the form of the time-dependent mass is. This calculation involves inserting the ansatz (5) into the action (4), expanding the result to second order in the fluctuating fields, making use of the background and of the constraint 
equations, and dropping total derivative terms from the action. In the context of scalar field matter, the quantum theory of cosmological fluctuations

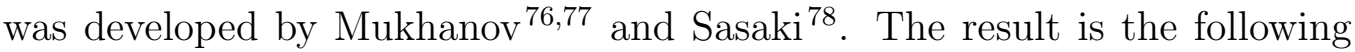
contribution $S^{(2)}$ to the action quadratic in the perturbations:

$$
S^{(2)}=\frac{1}{2} \int d^{4} x\left[v^{\prime 2}-v_{, i} v_{, i}+\frac{z^{\prime \prime}}{z} v^{2}\right],
$$

where the canonical variable $v$ (the "Sasaki-Mukhanov variable" introduced in $\frac{77}{\text { ) }}$ is given by

$$
v=a\left[\delta \varphi+\frac{\varphi_{0}^{\prime}}{\mathcal{H}} \Phi\right]
$$

with $\mathcal{H}=a^{\prime} / a$, and where

$$
z=\frac{a \varphi_{0}^{\prime}}{\mathcal{H}}
$$

As long as the equation of state does not change over time

$$
z(\eta) \sim a(\eta)
$$

Note that the variable $v$ is related to the curvature perturbation $\mathcal{R}$ in comoving coordinates introduced in ${ }^{79}$ and closely related to the variable $\zeta$ used in 80 :

$$
v=z \mathcal{R} .
$$

The equation of motion which follows from the action $(6)$ is (in momentum space)

$$
v_{k}^{\prime \prime}+k^{2} v_{k}-\frac{z^{\prime \prime}}{z} v_{k}=0,
$$

where $v_{k}$ is the k'th Fourier mode of $v$. The mass term in the above equation is in general given by the Hubble scale (the scale whose wave-number will be denoted $k_{H}$ ). Thus, it immediately follows that on small length scales, i.e. for $k>k_{H}$, the solutions for $v_{k}$ are constant amplitude oscillations . These oscillations freeze out at Hubble radius crossing, i.e. when $k=k_{H}$. On longer scales $\left(k \ll k_{H}\right)$, there is a mode of $v_{k}$ which scales as $z$. This mode is the dominant one in an expanding universe, but not in a contracting one.

Given the action (6), the cosmological perturbations can be quantized by canonical quantization (in the same way that a scalar matter field on a fixed cosmological background is quantized ${ }^{81}$ ). 
The final step in the quantum theory of cosmological perturbations is to specify an initial state. For an initial vacuum state we have harmonic oscillator ground state initial conditions for each Fourier mode of the field:

$$
\begin{aligned}
& v_{k}\left(\eta_{i}\right)=\frac{1}{\sqrt{2 k}} \\
& v_{k}^{\prime}\left(\eta_{i}\right)=\frac{\sqrt{k}}{\sqrt{2}}
\end{aligned}
$$

where $\eta_{i}$ is the conformal time corresponding to the initial physical time $t_{i}$.

In an expanding background, the scaling $v_{k} \sim z \sim a$ implies that the wave function of the quantum variable $v_{k}$ which performs quantum vacuum fluctuations on sub-Hubble scales, stops oscillating on super-Hubble scales and instead is squeezed (the amplitude increases in configuration space but decreases in momentum space). This squeezing corresponds to quantum particle production, and it is one of the two conditions which are required for the classicalization of the fluctuations 13 The second condition is decoherence which is induced by the non-linearities in the dynamical system which are inevitable since the Einstein action leads to highly nonlinear equations (see ${ }^{84}$ for an in-depth discussion of this point, and ${ }^{85}$ for related work). Note that the squeezing of cosmological fluctuations on super-Hubble scales occurs in all models, in particular in string gas cosmology and in the bouncing universe scenario since also in these scenarios perturbations propagate on super-Hubble scales for a long period of time. In a contracting phase, the dominant mode of $v_{k}$ on super-Hubble scales is also growing (the mode $v_{k} \sim a$ is now a decaying one). Thus, the squeezing of fluctuations on super-Hubble scales and the resulting classicalization of the perturbations also occurs.

\subsection{Fluctuations in Inflationary Cosmology}

In inflationary cosmology (see Fig. 1), we pick some initial time $t_{i}$ after the beginning of the inflationary phase and start with quantum vacuum initial conditions

$$
v_{k}\left(\eta_{i}\right)=\frac{1}{\sqrt{2 k}}
$$

\footnotetext{
${ }^{13}$ These conditions are necessary, but there is some debate as to whether they are sufficient to solve the "cosmic measurement problem" (see e.g. ${ }^{82}$ for an analysis from the physics perspective, and ${ }^{83}$ for a discussion in the framework of philosophy of physics).
} 
for all values of $k$ for which the wavelength is smaller than the Hubble radius at the initial time $t_{i}$. Since $v_{k}$ oscillates on sub-Hubble scales, the amplitude remains unchanged until the mode exits the Hubble radius at the time $t_{H}(k)$ given by

$$
a^{-1}\left(t_{H}(k)\right) k=H .
$$

We need to compute the power spectrum $\mathcal{P}_{\mathcal{R}}(k)$ of the curvature fluctuation $\mathcal{R}$ defined in 10 at some late time $t$ when the modes are super-Hubble. We first relate the power spectrum via the growth $v_{k} \sim a$ on super-Hubble scales to the power spectrum at the time $t_{H}(k)$ and then use the constancy of the amplitude of $v$ on sub-Hubble scales to relate it to the initial conditions (13). Thus

$$
\begin{aligned}
\mathcal{P}_{\mathcal{R}}(k, t) \equiv k^{3} \mathcal{R}_{k}^{2}(t) & =k^{3} z^{-2}(t)\left|v_{k}(t)\right|^{2} \\
& =k^{3} z^{-2}(t)\left(\frac{a(t)}{a\left(t_{H}(k)\right)}\right)^{2}\left|v_{k}\left(t_{H}(k)\right)\right|^{2} \\
& =k^{3} z^{-2}\left(t_{H}(k)\right)\left|v_{k}\left(t_{H}(k)\right)\right|^{2} \\
& \sim k^{3}\left(\frac{a(t)}{z(t)}\right)^{2} a^{-2}\left(t_{H}(k)\right)\left|v_{k}\left(t_{i}\right)\right|^{2}
\end{aligned}
$$

where in the final step we have used (9) and the constancy of the amplitude of $v$ on sub-Hubble scales. Making use of the condition (14) for Hubble radius crossing, and of the initial conditions (13), we immediately see that

$$
\mathcal{P}_{\mathcal{R}}(k, t) \sim\left(\frac{a(t)}{z(t)}\right)^{2} k^{3} k^{-2} k^{-1} H^{2},
$$

and that thus a scale invariant 14 power spectrum results. Since $H$ decreases slowly as inflation proceeds, the amplitude of the spectrum is slightly smaller for short wavelengths (which exit the Hubble radius later) than for longer ones. Thus, the spectrum has a slight tilt. Note that the alternative models which we discuss below also predict a slight red tilt of the spectrum. Current precision observations indicate that there indeed is a slight red tilt.

\subsection{Fluctuations in the Matter Bounce}

In the Matter Bounce paradigm (see Fig. 4) the universe starts out large but cold. Hence, it is reasonable to assume that fluctuations on cosmological

\footnotetext{
${ }^{14}$ Scale invariance means that the power spectrum is independent of $k$.
} 
scales begin in their vacuum state ${ }^{15}$. The vacuum spectrum is blue, i.e. there is more power on short wavelengths. To obtain a scale-invariant spectrum, a mechanism is required which boosts long wavelength modes relative to short wavelength ones. In a contracting universe long wavelength modes exit the Hubble radius earlier than short wavelength modes. If the fluctuation modes grow on super-Hubble scales, then we have a mechanism which boosts the long wavelength fluctuations.

In a contracting universe the mode $v_{k} \sim a$ is decaying and unimportant. The second mode, which is sub-dominant in an expanding universe, becomes the dominant one, and grows in time with a rate which depends on the equation of state of the background. It turns out that the growth rate in the case of a matter-dominated phase of contraction is exactly the correct one to turn an initial vacuum spectrum into a scale-invariant one, as was realized in $37 \sqrt[38]{ }$.

The analysis is not very complicated: if the contracting phase is matterdominated then $a(t) \sim t^{2 / 3}, \eta(t) \sim t^{1 / 3}$ and the dominant mode of $v$ scales as $\eta^{-1}$. Thus, the power spectrum of curvature fluctuations becomes

$$
\begin{aligned}
P_{\mathcal{R}}(k, \eta) & \sim k^{3}\left|v_{k}(\eta)\right|^{2} a^{-2}(\eta) \\
& \sim k^{3}\left|v_{k}\left(\eta_{H}(k)\right)\right|^{2}\left(\frac{\eta_{H}(k)}{\eta}\right)^{2} \sim k^{3-1-2} \\
& \sim \text { const }
\end{aligned}
$$

where we have made use of the assumption that we have a vacuum spectrum at Hubble radius crossing.

At this point we have shown that the spectrum of fluctuations is scaleinvariant on super-Hubble scales before the bounce phase. The evolution during the bounce depends in principle on the specific realization of the nonsingular bounce. In any concrete model, the equations of motion can be solved numerically without approximation during the bounce. Alternatively, we can solve them approximately using analytical techniques. The bottom line of a large number of studies in specific realizations of non-singular bouncing cosmologies (see e.g. $\frac{86[87 / 42 / 46 / 88 \mid 40}{15}$ is that on length scales large compared to the time of the bounce, the spectrum of curvature fluctuations is not

\footnotetext{
${ }^{15}$ The vacuum state of the fluctuation field $v$. This prescription does not require matter to be a quantum field. Cosmological perturbations can be quantized ${ }^{173}$ even if matter is a usual hydrodynamical fluid.
} 
changed during the bounce phase. Since typically the bounce time is set by a microphysical scale whereas the wavelength of fluctuations which we observe today is macroscopic (about $1 \mathrm{~mm}$ if the bounce scale is set by the particle physics GUT scale), we conclude that for scales relevant to current observations the spectrum is unchanged during the bounce. This completes the demonstration that a non-singular matter bounce leads to a scale-invariant spectrum of cosmological perturbations after the bounce provided that the initial spectrum on sub-Hubble scales is vacuum.

A specific signal of growing curvature fluctuations on super-Hubble scales in the contracting matter-dominated phase is a special form ${ }^{[89}$ of the "bispectrum", the three point correlation function of the CMB. The predicted amplitude of the bispectrum is also significantly larger than what is obtained in simple inflationary models. Thus, the way to observationally distinguish between the matter bounce and the inflationary paradigms is via precision observations of the bispectrum.

I close this section with a side comment which might be of interest to philosophers of cosmology: the fact that fluctuations grow both in the contracting and expanding phase has implications for attempts to build a cyclic cosmology. In four space-time dimensions it is impossible to construct a model which is cyclic in the presence of fluctuations - the growth of fluctuations breaks any cyclicity which may be present at the background level. Four space-time-dimensional cyclic background cosmologies are not predictive - the index of the power spectrum changes from cycle to cycle ${ }^{90}$. Note that the cyclic version of the Ekpyrotic scenario ${ }^{35}$ avoids these problems because it is not cyclic in the above sense: it is a higher space-time-dimensional model in which the radius of an extra dimension evolves cyclically, but the four-dimensional scale factor does not.

\subsection{Fluctuations in String Gas Cosmology}

In String Gas Cosmology (see Fig. 6) the physical wavelength of a fluctuation mode is constant in the Hagedorn phase since space is static. The Hubble radius is infinite in this phase. Hence, as in the case of inflationary cosmology, fluctuation modes begin sub-Hubble during the Hagedorn phase, and thus a causal generation mechanism for fluctuations is possible.

However, the physics of the generation mechanism is very different. In the case of inflationary cosmology, fluctuations are assumed to start as quantum vacuum perturbations because classical inhomogeneities are red-shifting. In 
contrast, in the Hagedorn phase of string gas cosmology there is no redshifting of classical matter. Hence, it is the fluctuations in the classical matter which dominate. Since classical matter is a string gas, the dominant fluctuations are string thermodynamic fluctuations. It turns out that the holographic scaling of the specific heat capacity of a gas of closed strings is key to obtaining a scale-invariant spectrum of curvature fluctuations.

The proposal for string gas structure formation is the following ${ }^{\sqrt[55]{5}}$ ( $\mathrm{sec}^{\sqrt{91}}$ for a more detailed description). For a fixed co-moving scale with wavenumber $k$ we compute the matter fluctuations of the thermal string gas, while the scale is sub-Hubble (and therefore gravitational effects are sub-dominant). When the scale exits the Hubble radius at time $t_{i}(k)$ we use the gravitational constraint equations to determine the induced metric fluctuations, which are then propagated to late times using the usual equations of gravitational perturbation theory. Since the scales we are interested in are in the far infrared, we use the Einstein constraint equations for fluctuations.

Assuming that the fluctuations are described by the perturbed Einstein equations (they are not if the dilaton is not fixed ${ }^{[92 \mid 93}$ ), then the spectra of cosmological perturbations $\Phi$ and gravitational waves $h$ are given by the energy-momentum fluctuations in the following way ${ }^{91}$

$$
\left\langle|\Phi(k)|^{2}\right\rangle=16 \pi^{2} G^{2} k^{-4}\left\langle\delta T_{0}^{0}(k) \delta T_{0}^{0}(k)\right\rangle,
$$

where the pointed brackets indicate expectation values, and

$$
\left\langle|h(k)|^{2}\right\rangle=16 \pi^{2} G^{2} k^{-4}\left\langle\delta T^{i}{ }_{j}(k) \delta T^{i}{ }_{j}(k)\right\rangle,
$$

where on the right hand side of $(19)$ we mean the average over the correlation functions with $i \neq j$, and $h$ is the amplitude of the gravitational waves ${ }^{16}$.

The equation (18) is used to determine the spectrum of scalar metric fluctuations. We first calculate the root mean square energy density fluctuations in a sphere of radius $R=k^{-1}$. For a system in thermal equilibrium they are given by the specific heat capacity $C_{V}$ via

$$
\left\langle\delta \rho^{2}\right\rangle=\frac{T^{2}}{R^{6}} C_{V} .
$$

\footnotetext{
${ }^{16}$ The gravitational wave tensor $h_{i j}$ can be written as the amplitude $h$ multiplied by a constant polarization tensor.
} 
The specific heat of a gas of closed strings on a torus of radius $R$ can be derived from the partition function of a gas of closed strings. This computation was carried out in ${ }^{94}$ (see also ${ }^{95}$ ) with the result

$$
C_{V} \approx 2 \frac{R^{2} / \ell^{3}}{T\left(1-T / T_{H}\right)} .
$$

The specific heat capacity scales holographically with the size of the box. This result follows rigorously from evaluating the string partition function in the Hagedorn phase. The result, however, can also be understood heuristically: in the Hagedorn phase the string winding modes are crucial. These modes look like point particles in one less spatial dimension. Hence, we expect the specific heat capacity to scale like in the case of point particles in one less dimension of space ${ }^{17}$.

With these results, the power spectrum $P(k)$ of scalar metric fluctuations can be evaluated as follows

$$
\begin{aligned}
P_{\Phi}(k) & \equiv \frac{1}{2 \pi^{2}} k^{3}|\Phi(k)|^{2} \\
& =8 G^{2} k^{-1}<|\delta \rho(k)|^{2}>. \\
& =8 G^{2} k^{2}<(\delta M)^{2}>_{R} \\
& =8 G^{2} k^{-4}<(\delta \rho)^{2}>_{R} \\
& =8 G^{2} \frac{T}{\ell_{s}^{3}} \frac{1}{1-T / T_{H}},
\end{aligned}
$$

where in the first step we have used (18) to replace the expectation value of $|\Phi(k)|^{2}$ in terms of the correlation function of the energy density, and in the second step we have made the transition to position space.

The first conclusion from the result 22 is that the spectrum is approximately scale-invariant $(P(k)$ is independent of $k)$. It is the 'holographic' scaling $C_{V}(R) \sim R^{2}$ which is responsible for the overall scale-invariance of the spectrum of cosmological perturbations. However, there is a small $k$ dependence which comes from the fact that in the above equation for a scale $k$ the temperature $T$ is to be evaluated at the time $t_{i}(k)$. Thus, the factor $\left(1-T / T_{H}\right)$ in the denominator is responsible for giving the spectrum a slight

\footnotetext{
${ }^{17}$ We emphasize that it was important for us to have compact spatial dimensions in order to obtain the winding modes which are crucial to get the holographic scaling of the thermodynamic quantities.
} 
dependence on $k$. Since the temperature slightly decreases as time increases at the end of the Hagedorn phase, shorter wavelengths for which $t_{i}(k)$ occurs later obtain a smaller amplitude. Thus, the spectrum has a slight red tilt.

As discovered in ${ }^{96}$, the spectrum of gravitational waves is also nearly scale invariant. However, in the expression for the spectrum of gravitational waves the factor $\left(1-T / T_{H}\right)$ appears in the numerator, thus leading to a slight blue tilt in the spectrum. This is a prediction with which the cosmological effects of string gas cosmology can be distinguished from those of inflationary cosmology, where quite generically a slight red tilt for gravitational waves results. The physical reason for the blue tilt in string gas cosmology is that large scales exit the Hubble radius earlier when the pressure and hence also the off-diagonal spatial components of $T_{\mu \nu}$ are closer to zero. For more details the interested reader is referred to the original literature.

\section{Discussion}

As I hope to have convinced the reader, inflationary cosmology, in spite of its spectacular phenomenological success, suffers from several conceptual problems and should at this point not yet be considered as an established theory of the early universe. The recent speculations concerning the "inflationary multiverse" face even more serious problems and should be viewed with caution.

I have presented two alternative scenarios which are in equally good agreement with current observations on the distribution of galaxies and anisotropies of the CMB as the inflationary paradigm ${ }^{18}$ These two scenarios make predictions for future observations which are different from those of inflation. Which of these scenarios is actually realized in nature will ultimately be determined by observations.

The two alternative scenarios which I focused on are the Matter Bounce paradigm involving a non-singular background cosmology which starts in a matter-dominated phase of contraction, and the Emergent Universe picture as realized in String Gas Cosmology. These are two scenarios the author has

\footnotetext{
${ }^{18}$ At this point it must be emphasized again that obtaining a scale-invariant spectrum of curvature fluctuations is a very non-trivial requirement for early universe cosmology. For example, initial vacuum fluctuations generated in a contracting universe are not scaleinvariant unless the relevant phase of matter contraction is dominated by non-relativistic matter.
} 
worked on. However, there are other scenarios, a particularly promising one being the Ekpyrotic scenario ${ }^{34}$, which also leads to a picture in which time runs from $-\infty$ to $+\infty$.

None of the alternative scenarios are without problems. In fact, one may argue that none of them address all of the classic problems of Standard Big Bang cosmology as well as inflation does. The matter bounce scenario suffers from an instability to the development of anisotropies, and emergent scenarios do not as nicely explain the large size and entropy of the universe as inflation does. However, both of them are free from one of the key problems of inflationary cosmology, namely the trans-Planckian problem for cosmological perturbations.

Acknowledgements: I wish to thank Henrik Zinkernagel for the invitation to participate and lecture at this stimulating workshop, and also for useful comments on the draft of these lecture notes. I wish to thank all of my collaborators on whose work I have drawn. This work has been supported in part by funds from an NSERC Discovery Grant and from the Canada Research

Chair program. I also acknowledge support from the Killam Foundation for the period $9 / 09-8 / 11$.

\section{References}

[1] Guth AH, "The Inflationary Universe: A Possible Solution To The Horizon And Flatness Problems," Phys. Rev. D 23, 347 (1981).

[2] R. Brout, F. Englert and E. Gunzig, "The Creation Of The Universe As A Quantum Phenomenon," Annals Phys. 115, 78 (1978).

[3] A. A. Starobinsky, "A New Type Of Isotropic Cosmological Models Without Singularity," Phys. Lett. B 91, 99 (1980).

[4] K. Sato, "First Order Phase Transition Of A Vacuum And Expansion Of The Universe," Mon. Not. Roy. Astron. Soc. 195, 467 (1981).

[5] V. Mukhanov and G. Chibisov, "Quantum Fluctuation And Nonsingular Universe. (In Russian)," JETP Lett. 33, 532 (1981) [Pisma Zh. Eksp. Teor. Fiz. 33, 549 (1981)].

[6] W. Press, "Spontaneous production of the Zel'dovich spectrum of cosmological fluctuations", Phys. Scr. 21, 702 (1980). 
[7] C. L. Bennett et al., "First Year Wilkinson Microwave Anisotropy Probe (WMAP) Observations: Preliminary Maps and Basic Results," Astrophys. J. Suppl. 148, 1 (2003) arXiv:astro-ph/0302207|.

[8] R. A. Sunyaev, Y. .B. Zeldovich, "Small scale fluctuations of relic radiation," Astrophys. Space Sci. 7, 3-19 (1970).

[9] P. J. E. Peebles, J. T. Yu, "Primeval adiabatic perturbation in an expanding universe," Astrophys. J. 162, 815-836 (1970).

[10] G. F. Smoot, C. L. Bennett, A. Kogut, E. L. Wright, J. Aymon, N. W. Boggess, E. S. Cheng and G. De Amici et al., "Structure in the COBE differential microwave radiometer first year maps," Astrophys. J. 396, L1 (1992).

[11] P. D. Mauskopf et al. [Boomerang Collaboration], "Measurement of a peak in the cosmic microwave background power spectrum from the North American test flight of BOOMERANG," Astrophys. J. 536, L59 (2000) astro-ph/9911444.

[12] A. D. Linde, "Chaotic Inflation," Phys. Lett. B 129, 177 (1983).

[13] R. H. Brandenberger and J. H. Kung, "Chaotic Inflation As An Attractor In Initial Condition Space," Phys. Rev. D 42, 1008 (1990);

R. H. Brandenberger, H. Feldman and J. Kung, "Initial conditions for chaotic inflation," Phys. Scripta T 36, 64 (1991).

[14] F. L. Bezrukov and M. Shaposhnikov, "The Standard Model Higgs boson as the inflaton," Phys. Lett. B 659, 703 (2008) arXiv:0710.3755 [hepth]].

[15] A. D. Linde, "Inflationary Cosmology," Lect. Notes Phys. 738, 1 (2008) arXiv:0705.0164 [hep-th]];

A. Mazumdar and J. Rocher, "Particle physics models of inflation and curvaton scenarios," Phys. Rept. 497, 85 (2011) arXiv:1001.0993 [hep$\mathrm{ph}]$.

[16] A. A. Starobinsky, "Stochastic De Sitter (inflationary) Stage In The Early Universe," In *De Vega, H.j. ( Ed.), Sanchez, N. ( Ed.): Field Theory, Quantum Gravity and Strings*, 107-126 
[17] A. Vilenkin, "The Birth of Inflationary Universes," Phys. Rev. D 27, 2848 (1983);

A. D. Linde, "Eternally Existing Selfreproducing Chaotic Inflationary Universe," Phys. Lett. B 175, 395 (1986).

[18] F. Denef and M. R. Douglas, "Distributions of flux vacua," JHEP 0405, 072 (2004) hep-th/0404116.

[19] Universe or Multiverse?, B. Carr ed., Cambridge University Press (2007),

[20] H. Ooguri and C. Vafa, "On the Geometry of the String Landscape and the Swampland," Nucl. Phys. B 766, 21 (2007) [hep-th/0605264].

[21] V. F. Mukhanov, L. R. W. Abramo and R. H. Brandenberger, "On the Back reaction problem for gravitational perturbations," Phys. Rev. Lett. 78, 1624 (1997) gr-qc/9609026];

R. H. Brandenberger, "Back reaction of cosmological perturbations and the cosmological constant problem," hep-th/0210165.

[22] F. C. Adams, K. Freese and A. H. Guth, "Constraints on the scalar field potential in inflationary models," Phys. Rev. D 43, 965 (1991).

[23] R. H. Brandenberger, "Inflationary cosmology: Progress and problems," arXiv:hep-ph/9910410.

[24] N. Kaloper, M. Kleban, A. E. Lawrence and S. Shenker, "Signatures of short distance physics in the cosmic microwave background," Phys. Rev. D 66, 123510 (2002) [hep-th/0201158].

[25] R. H. Brandenberger and J. Martin, "The Robustness of inflation to changes in superPlanck scale physics," Mod. Phys. Lett. A 16, 999 (2001), arXiv:astro-ph/0005432;

J. Martin and R. H. Brandenberger, "The TransPlanckian problem of inflationary cosmology," Phys. Rev. D 63, 123501 (2001), arXiv:hepth/0005209.

[26] J. C. Niemeyer, "Inflation with a high frequency cutoff," Phys. Rev. D 63, 123502 (2001), arXiv:astro-ph/0005533];

J. C. Niemeyer and R. Parentani, "Minimal modifications of the primordial power spectrum from an adiabatic short distance cutoff," 
Phys. Rev. D 64, 101301 (2001), arXiv:astro-ph/0101451;

S. Shankaranarayanan, "Is there an imprint of Planck scale physics on inflationary cosmology?," Class. Quant. Grav. 20, 75 (2003) [arXiv:gr-qc/0203060].

[27] R. Brandenberger and X. -m. Zhang, "The Trans-Planckian Problem for Inflationary Cosmology Revisited," arXiv:0903.2065 [hep-th].

[28] S. W. Hawking and G. F. R. Ellis, "The Large scale structure of spacetime," Cambridge University Press, Cambridge, 1973

[29] A. Borde and A. Vilenkin, "Eternal inflation and the initial singularity," Phys. Rev. Lett. 72, 3305 (1994) arXiv:gr-qc/9312022.

[30] H. Zinkernagel, "Did Time Have a Beginning?", Int. Studies in the Philosophy of Science 22, 237 (2008).

[31] M. Novello and S. E. P. Bergliaffa, "Bouncing Cosmologies," Phys. Rept. 463, 127 (2008) [arXiv:0802.1634 [astro-ph]].

[32] M. Gasperini and G. Veneziano, "Pre - big bang in string cosmology," Astropart. Phys. 1, 317 (1993) [arXiv:hep-th/9211021].

[33] R. H. Brandenberger and C. Vafa, "Superstrings in the Early Universe," Nucl. Phys. B 316, 391 (1989).

[34] J. Khoury, B. A. Ovrut, P. J. Steinhardt and N. Turok, "The ekpyrotic universe: Colliding branes and the origin of the hot big bang," Phys. Rev. D 64, 123522 (2001) arXiv:hep-th/0103239.

[35] P. J. Steinhardt and N. Turok, "Cosmic evolution in a cyclic universe," Phys. Rev. D 65, 126003 (2002) [hep-th/0111098].

[36] T. J. Battefeld, S. P. Patil and R. Brandenberger, "Non-singular perturbations in a bouncing brane model," Phys. Rev. D 70, 066006 (2004) hep-th/0401010].

[37] D. Wands, "Duality invariance of cosmological perturbation spectra," Phys. Rev. D 60, 023507 (1999) arXiv:gr-qc/9809062]. 
[38] F. Finelli and R. Brandenberger, "On the generation of a scaleinvariant spectrum of adiabatic fluctuations in cosmological models with a contracting phase," Phys. Rev. D 65, 103522 (2002) arXiv:hepth/0112249.

[39] C. Kounnas, H. Partouche and N. Toumbas, "Thermal duality and nonsingular cosmology in d-dimensional superstrings," Nucl. Phys. B 855, 280 (2012) arXiv:1106.0946 [hep-th]];

C. Kounnas, H. Partouche and N. Toumbas, "S-brane to thermal nonsingular string cosmology," arXiv:1111.5816 [hep-th].

[40] R. Brandenberger, C. Kounnas, H. Partouche, S. Patil and N. Toumbas, "Fluctuations in Non-Singular Bouncing Cosmologies from Type II Superstrings", to be submitted.

[41] B. Feng, X. L. Wang and X. M. Zhang, "Dark Energy Constraints from the Cosmic Age and Supernova," Phys. Lett. B 607, 35 (2005) arXiv:astro-ph/0404224];

B. Feng, M. Li, Y. S. Piao and X. Zhang, "Oscillating quintom and the recurrent universe," Phys. Lett. B 634, 101 (2006) arXiv:astroph/0407432; ;

Y. F. Cai, T. Qiu, Y. S. Piao, M. Li and X. Zhang, "Bouncing Universe with Quintom Matter," JHEP 0710, 071 (2007) arXiv:0704.1090 [grqc]];

Y. F. Cai, T. T. Qiu, J. Q. Xia and X. Zhang, "A Model Of Inflationary Cosmology Without Singularity," Phys. Rev. D 79, 021303 (2009) arXiv:0808.0819 [astro-ph]].

[42] Y. F. Cai, T. Qiu, R. Brandenberger, Y. S. Piao and X. Zhang, "On Perturbations of Quintom Bounce," JCAP 0803, 013 (2008) arXiv:0711.2187 [hep-th]];

Y. F. Cai and X. Zhang, "Evolution of Metric Perturbations in Quintom Bounce model," JCAP 0906, 003 (2009) arXiv:0808.2551 [astro-ph]];

Y. F. Cai, T. Qiu, R. Brandenberger and X. Zhang, "A Nonsingular Cosmology with a Scale-Invariant Spectrum of Cosmological Perturbations from Lee-Wick Theory," Phys. Rev. D 80, 023511 (2009) arXiv:0810.4677 [hep-th]].

[43] R. H. Brandenberger, "Alternatives to Cosmological Inflation," arXiv:0902.4731 [hep-th]; 
R. H. Brandenberger, "Cosmology of the Very Early Universe," AIP Conf. Proc. 1268, 3-70 (2010). [arXiv:1003.1745 [hep-th]];

R. H. Brandenberger, "Introduction to Early Universe Cosmology," PoS ICFI 2010, 001 (2010) arXiv:1103.2271 [astro-ph.CO]].

[44] J. M. Cline, S. Jeon and G. D. Moore, "The phantom menaced: Constraints on low-energy effective ghosts," Phys. Rev. D 70, 043543 (2004) arXiv:hep-ph/0311312.

[45] P. Creminelli and L. Senatore, "A smooth bouncing cosmology with scale invariant spectrum," JCAP 0711, 010 (2007) arXiv:hep-th/0702165];

E. I. Buchbinder, J. Khoury and B. A. Ovrut, "New Ekpyrotic Cosmology," Phys. Rev. D 76, 123503 (2007) arXiv:hep-th/0702154.

[46] C. Lin, R. H. Brandenberger and L. P. Levasseur, "A Matter Bounce By Means of Ghost Condensation," JCAP 1104, 019 (2011) arXiv:1007.2654 [hep-th]].

[47] T. Qiu, J. Evslin, Y. F. Cai, M. Li and X. Zhang, "Bouncing Galileon Cosmologies," JCAP 1110, 036 (2011) [arXiv:1108.0593 [hep-th]];

D. A. Easson, I. Sawicki and A. Vikman, "G-Bounce," JCAP 1111, 021 (2011) arXiv:1109.1047 [hep-th]].

[48] R. H. Brandenberger, V. F. Mukhanov and A. Sornborger, "A Cosmological theory without singularities," Phys. Rev. D 48, 1629 (1993) [arXiv:gr-qc/9303001].

[49] T. Biswas, A. Mazumdar and W. Siegel, "Bouncing universes in stringinspired gravity," JCAP 0603, 009 (2006) arXiv:hep-th/0508194.

[50] R. Brandenberger, "Matter Bounce in Horava-Lifshitz Cosmology," Phys. Rev. D 80, 043516 (2009) arXiv:0904.2835 [hep-th]].

[51] P. Horava, "Quantum Gravity at a Lifshitz Point," Phys. Rev. D 79, 084008 (2009) [arXiv:0901.3775 [hep-th]].

[52] M. Bojowald, "Quantum Cosmology," Lect. Notes Phys. 835, 1 (2011); A. Ashtekar and P. Singh, "Loop Quantum Cosmology: A Status Report," Class. Quant. Grav. 28, 213001 (2011) arXiv:1108.0893 [gr-qc]]. 
[53] G. F. R. Ellis and R. Maartens, "The emergent universe: Inflationary cosmology with no singularity," Class. Quant. Grav. 21, 223 (2004) grqc/0211082 ;

G. F. R. Ellis, J. Murugan and C. G. Tsagas, "The Emergent universe: An Explicit construction," Class. Quant. Grav. 21, 233 (2004) gr-qc/0307112.

[54] T. Biswas, R. Brandenberger, A. Mazumdar and W. Siegel, "Nonperturbative gravity, Hagedorn bounce and CMB," JCAP 0712, 011 (2007) arXiv:hep-th/0610274.

[55] A. Nayeri, R. H. Brandenberger and C. Vafa, "Producing a scaleinvariant spectrum of perturbations in a Hagedorn phase of string cosmology," Phys. Rev. Lett. 97, 021302 (2006) arXiv:hep-th/0511140.

[56] J. Kripfganz and H. Perlt, "Cosmological Impact Of Winding Strings," Class. Quant. Grav. 5, 453 (1988).

[57] R. H. Brandenberger, "String Gas Cosmology," arXiv:0808.0746 [hepth].

[58] T. Battefeld and S. Watson, "String gas cosmology," Rev. Mod. Phys. 78, 435 (2006) arXiv:hep-th/0510022.

[59] R. Hagedorn, "Statistical Thermodynamics Of Strong Interactions At High-Energies," Nuovo Cim. Suppl. 3, 147 (1965).

[60] J. Polchinski, String Theory, Vols. 1 and 2, (Cambridge Univ. Press, Cambridge, 1998).

[61] T. Boehm and R. Brandenberger, "On T-duality in brane gas cosmology," JCAP 0306, 008 (2003) arXiv:hep-th/0208188.

[62] M. Sakellariadou, "Numerical Experiments in String Cosmology," Nucl. Phys. B 468, 319 (1996) arXiv:hep-th/9511075.

[63] G. B. Cleaver and P. J. Rosenthal, "String cosmology and the dimension of space-time," Nucl. Phys. B 457, 621 (1995) arXiv:hep-th/9402088.

[64] R. Easther, B. R. Greene, M. G. Jackson and D. Kabat, "String windings in the early universe," JCAP 0502, 009 (2005) arXiv:hep-th/0409121. 
[65] R. Danos, A. R. Frey and A. Mazumdar, "Interaction rates in string gas cosmology," Phys. Rev. D 70, 106010 (2004) arXiv:hep-th/0409162.

[66] B. Greene, D. Kabat and S. Marnerides, "Dynamical Decompactification and Three Large Dimensions," arXiv:0908.0955 [hep-th].

[67] S. Watson and R. Brandenberger, "Stabilization of extra dimensions at tree level," JCAP 0311, 008 (2003) |arXiv:hep-th/0307044|;

S. Watson, "Moduli stabilization with the string Higgs effect," Phys. Rev. D 70, 066005 (2004) arXiv:hep-th/0404177.

[68] S. P. Patil and R. Brandenberger, "Radion stabilization by stringy effects in general relativity and dilaton gravity," Phys. Rev. D 71, 103522 (2005) [arXiv:hep-th/0401037.

[69] S. P. Patil and R. H. Brandenberger, "The cosmology of massless string modes," JCAP 0601, 005 (2006) [arXiv:hep-th/0502069].

[70] R. Brandenberger, Y. K. Cheung and S. Watson, "Moduli stabilization with string gases and fluxes," JHEP 0605, 025 (2006) arXiv:hepth/0501032.

[71] R. J. Danos, A. R. Frey and R. H. Brandenberger, "Stabilizing moduli with thermal matter and nonperturbative effects," Phys. Rev. D 77, 126009 (2008) arXiv:0802.1557 [hep-th]];

S. Mishra, W. Xue, R. Brandenberger and U. Yajnik, "Supersymmetry Breaking and Dilaton Stabilization in String Gas Cosmology," arXiv:1103.1389 [hep-th].

[72] R. H. Brandenberger, "Lectures on the theory of cosmological perturbations," Lect. Notes Phys. 646, 127 (2004) arXiv:hep-th/0306071].

[73] V. F. Mukhanov, H. A. Feldman and R. H. Brandenberger, "Theory of cosmological perturbations. Part 1. Classical perturbations. Part 2. Quantum theory of perturbations. Part 3. Extensions," Phys. Rept. 215, 203 (1992).

[74] A. Berera, "Warm inflation," Phys. Rev. Lett. 75, 3218 (1995) astroph/9509049. 
[75] A. A. Starobinsky, "Spectrum of relict gravitational radiation and the early state of the universe," JETP Lett. 30, 682 (1979) [Pisma Zh. Eksp. Teor. Fiz. 30, 719 (1979)].

[76] V. F. Mukhanov, "Quantum Theory of Gauge Invariant Cosmological Perturbations," Sov. Phys. JETP 67, 1297 (1988) [Zh. Eksp. Teor. Fiz. 94N7, 1 (1988)].

[77] V. F. Mukhanov, "Gravitational Instability Of The Universe Filled With A Scalar Field," JETP Lett. 41, 493 (1985) [Pisma Zh. Eksp. Teor. Fiz. 41, 402 (1985)].

[78] M. Sasaki, "Large Scale Quantum Fluctuations in the Inflationary Universe," Prog. Theor. Phys. 76, 1036 (1986).

[79] D. H. Lyth, "Large Scale Energy Density Perturbations And Inflation," Phys. Rev. D 31, 1792 (1985).

[80] J. M. Bardeen, P. J. Steinhardt and M. S. Turner, "Spontaneous Creation Of Almost Scale - Free Density Perturbations In An Inflationary Universe," Phys. Rev. D 28, 679 (1983);

R. H. Brandenberger and R. Kahn, "Cosmological Perturbations In Inflationary Universe Models," Phys. Rev. D 29, 2172 (1984).

[81] N. D. Birrell and P. C. W. Davies, "Quantum Fields In Curved Space," Cambridge, Uk: Univ. Pr. (1982) 340p

[82] D. Sudarsky, "Shortcomings in the Understanding of Why Cosmological Perturbations Look Classical," Int. J. Mod. Phys. D 20, 509 (2011) arXiv:0906.0315 [gr-qc]].

[83] H. Zinkernagel, "Some Trends in the Philosophy of Physics", Theoria 71, 215 (2011);

S. Rugh and H. Zinkernagel, "Weyl's Principle: Cosmic Time and Quantum Fundamentalism", in D. Dirks et al. (eds), Explanation, Prediction and Confirmation, The Philosophy of Science in a European Perspective 2, DOI 10.1007/978-94-007-1180-8_28 (Springer, 2011).

[84] C. Kiefer, I. Lohmar, D. Polarski and A. A. Starobinsky, "Pointer states for primordial fluctuations in inflationary cosmology," Class. Quant. Grav. 24, 1699 (2007) arXiv:astro-ph/0610700]. 
[85] P. Martineau, "On the decoherence of primordial fluctuations during inflation," Class. Quant. Grav. 24, 5817 (2007) [arXiv:astro-ph/0601134].

[86] S. Alexander, T. Biswas and R. H. Brandenberger, "On the Transfer of Adiabatic Fluctuations through a Nonsingular Cosmological Bounce," arXiv:0707.4679 [hep-th].

[87] R. Brandenberger, H. Firouzjahi and O. Saremi, "Cosmological Perturbations on a Bouncing Brane," JCAP 0711, 028 (2007) arXiv:0707.4181 [hep-th]].

[88] X. Gao, Y. Wang, W. Xue and R. Brandenberger, "Fluctuations in a Hořava-Lifshitz Bouncing Cosmology," JCAP 1002, 020 (2010) arXiv:0911.3196 [hep-th]];

X. Gao, Y. Wang, R. Brandenberger and A. Riotto, "Cosmological Perturbations in Hořava-Lifshitz Gravity," Phys. Rev. D 81, 083508 (2010) arXiv:0905.3821 [hep-th]].

[89] Y. -F. Cai, W. Xue, R. Brandenberger and X. Zhang, "Non-Gaussianity in a Matter Bounce," JCAP 0905, 011 (2009) arXiv:0903.0631 [astroph.CO]].

[90] R. H. Brandenberger, "Processing of Cosmological Perturbations in a Cyclic Cosmology," Phys. Rev. D 80, 023535 (2009) arXiv:0905.1514 [hep-th]].

[91] R. H. Brandenberger, A. Nayeri, S. P. Patil and C. Vafa, "String gas cosmology and structure formation," Int. J. Mod. Phys. A 22, 3621 (2007) arXiv:hep-th/0608121.

[92] R. H. Brandenberger et al., "More on the spectrum of perturbations in string gas cosmology," JCAP 0611, 009 (2006) arXiv:hep-th/0608186.

[93] N. Kaloper, L. Kofman, A. Linde and V. Mukhanov, "On the new string theory inspired mechanism of generation of cosmological perturbations," JCAP 0610, 006 (2006) arXiv:hep-th/0608200.

[94] N. Deo, S. Jain, O. Narayan and C. I. Tan, "The Effect of topology on the thermodynamic limit for a string gas," Phys. Rev. D 45, 3641 (1992). 
[95] A. Nayeri, "Inflation free, stringy generation of scale-invariant cosmological fluctuations in $\mathrm{D}=3+1$ dimensions," arXiv:hep-th/0607073.

[96] R. H. Brandenberger, A. Nayeri, S. P. Patil and C. Vafa, "Tensor modes from a primordial Hagedorn phase of string cosmology," Phys. Rev. Lett. 98, 231302 (2007) |arXiv:hep-th/0604126|. 